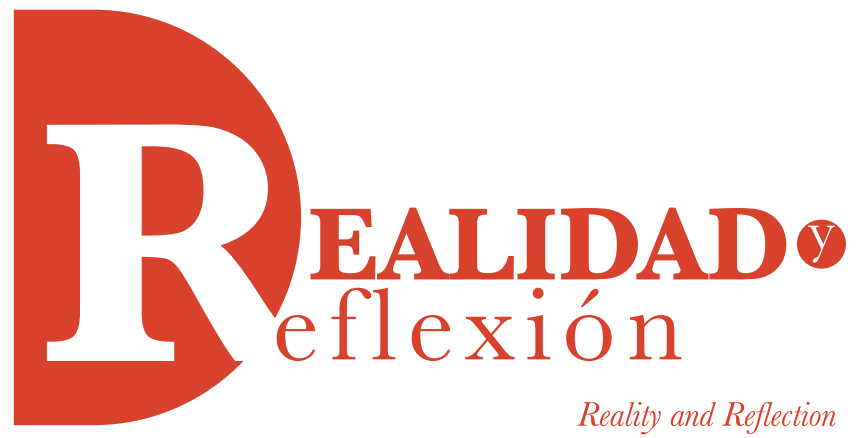

ISSN 1992-6510

e-ISSN 2520-92990

Año 17, N 45, San Salvador, El Salvador, Centroamérica. Revista Semestral Enero-Junio 2017

YEAR 17, N 45, SAN SALVADOR, EL SALVADOR, CENTRAL AMERICA. SEMESTRAL JOURNAL JANUARY-JUNE 2017

\title{
Pedagogía en centros penitenciarios latinoamericanos: Una realidad invisibilizada
}

\author{
Pedagogy in Latin American Penitentiary Institutions: \\ A Hidden Reality
}

Carolina Elizabeth San Lucas Solórzano Universidad Técnica de Ambato, Facultad de Ciencias Humanas y de la Educación, Carrera De Educación Parvularia Licenciada en Ciencias de la Educación, Mención Educación Parvularia Universidad Técnica de Ambato Doctora en Ciencias de la Educación, Mención Gerencia Educativa Universidad Técnica de Ambato Magister en Gerencia y Mediación de Centros Educativos Infantiles Universidad Técnica de Ambato carolinaesanlucass@uta.edu.ec

Elena Del Rocío Rosero Morales Universidad Técnica de Ambato, Facultad de Ciencias Humanas y de la Educación, Carrera De Educación Parvularia Psicóloga Educativa y Orientadora Vocacional Universidad Técnica de Ambato Magister en Psicología Educativa Universidad Técnica de Ambato elenadroserom@uta.edu.ec

Sylvia Andrade Universidad Técnica de Ambato, Facultad de Ciencias Humanas y de la Educación, Carrera De Educación Parvularia Licenciada en Ciencias de la Educación, Mención Educación Parvularia Universidad Técnica de Ambato

Doctora en Ciencias de la Educación, Mención Gerencia Educativa Universidad Técnica de Ambato Magister en Docencia Universitaria y Administración Educativa Universidad Tecnológica Indoamerica sylviajandradez@uta.edu.ec

Fecha de recepción: 27 de enero de 2017 Fecha de aprobación: 30 de abril de 2017 


\title{
RESUMEN
}

Un recorrido teórico legal a nivel latinoamericano muestra el interés y los esfuerzos sumados por la realidad penitenciaria de cada país en cumplimiento de los derechos humanos. La adecuada reinserción social que disminuya la reincidencia en actos delictivos de la sociedad es el objetivo primordial; la práctica pedagógica en el proceso de reinserción y promulgada en los modelos penitenciarios, visibiliza por un lado la intención de gobiernos en mejora del sistema, y por otro lado invisibiliza componentes que no ejecutoriados adecuadamente por factores como el contexto de encierro, la escasa capacitación de quienes están inmersos en el sistema y el olvido social, deteriora las posibilidades de una reinserción idónea de las personas privadas de libertad. Los estudios evidencian modelos penitenciarios con base en una pedagogía social comunitaria aplicados con éxito en ciertos contextos sociales, derivando la necesidad de que su implementación se dé bajo análisis técnicos, permitiendo su aplicabilidad en otras sociedades con igual o mayor apremio de cambio estructural en pro de una eficiente reinserción. El artículo contempla el método de síntesis del conocimiento científico, con una revisión de tipo narrativo basado en técnicas de rastreo y lectura de bibliografía referenciada.

Palabras clave: modelo penitenciario, práctica pedagógica, reinserción social, centros penitenciarios, educación en contextos de encierro.

\begin{abstract}
A theoretical and legal overview at the Latin American level shows that there is an interest in the current realities of penitentiary institutions, as well as in the efforts that have been made by each country in the region to meet human rights' needs. Adequate social reinsertion that lowers new incidences of criminal activity in society is the primordial objective. Moreover, the pedagogical practices carried out during the process of reinsertion into society, which are promoted by penitentiary models, highlights, on the one hand, the intention of governments to improve penitentiary systems. On the other hand, there are many hidden aspects that hinder the understanding of penitentiaries-many of which are not adequately executed. These are due to factors such as the context of incarceration itself, the lack of training of those individuals who are immersed in the system, and the social void left after incarceration, all of which reduce the possibilities of an ideal reinsertion into society. Current studies show, however, that there are penitentiary models rooted in communitybased social pedagogies, which have been applied successfully in certain social contexts, i.e. where there is a need for their implementation using technical analysis, thereby permitting their applicability in other societies with the same or greater urgency for structural change and promoting a more efficient reinsertion. This article considers the method of synthesis of scientific knowledge with a narrative-type vision, which is based on observation techniques and the reading of related bibliographical materials.
\end{abstract}

Keywords: penitentiary model, pedagogical practice, social reinsertion, penitentiary institutions, education within the context of incarceration.

\section{Argumento legal}

Hablar de educación en el Sistema de Rehabilitación Social implica un recorrido teórico que explique su evolución y estado actual en los mismos. Los principios, marco legal, normativas internacionales y nacionales dan cuenta de la importancia de una rehabilitación e inserción de las personas privadas de libertad en base a procesos pedagógicos profundos que impacten no solo en el momento sino en la permanencia ejecutada de un plan de vida.

Es así que la Organización de Naciones Unidas, (1977) aprueba las Reglas Mínimas para el tratamiento de los Reclusos expresando en su apartado: 77.1) Se tomarán disposiciones para mejorar la instrucción de todos los reclusos... la instrucción de los analfabetos y la de los reclusos jóvenes será obligatoria... 2) La instrucción deberá coordinarse con el sistema de instrucción pública a fin de que al ser puesto en libertad puedan continuar sin dificultad su preparación.

De igual manera los principios básicos para el tratamiento de los reclusos (ONU, 1990) manifiesta "Todos los reclusos tendrán derecho a participar en actividades culturales y educativas 
encaminadas a desarrollar plenamente la personalidad humana”. La (OEA, 2008) reitera en su principio XIII que “...Las personas privadas de libertad tendrán derecho a la educación, la cual será accesible para todas las personas, sin discriminación alguna, y tomará en cuenta la diversidad cultural y sus necesidades especiales”.

Son varias las instancias legales que corroboran el derecho a la educación de la persona privada de libertad, sin embargo es necesario resaltar hacia dónde van los objetivos educativos del Sistema de Rehabilitación Social, ante lo cual los Ministros de Justicia y representantes de los Estados Iberoamericanos reunidos en México recomiendan en la Declaración de San José (2010):

3) Generar las acciones necesarias para estimular que las personas penalmente privadas de libertad puedan utilizar ese tiempo en la adquisición de nuevas habilidades y competencias útiles para su desarrollo y para una mejor convivencia social, especialmente en materia de educación y trabajo, así como para su retorno a la comunidad.

8) Atender la necesidad de adoptar mecanismos adecuados para la progresiva incorporación al medio libre de las personas penalmente privadas de libertad, mediante solturas anticipadas al cumplimiento de la condena, con el fin de generar las mejores condiciones posibles para el retorno a la vida en libertad (CONJIB, 2010).

Fundamentado en los objetivos, normativas y declaraciones internacionales que defienden el derecho a la educación y procesos de inserción de las personas privadas de libertad, los países consolidan sus políticas de Estado; así el Ecuador no es la excepción, en el 2007 se declara en emergencia al sistema penitenciario ecuatoriano para la construcción de nuevos centros de privación de libertad así como la adecuación de los existentes y la implementación de un modelo de atención integral destinado a la rehabilitación social (SENPLADES, 2016), también contempla en la Constitución de la República del Ecuador:

Art. 51. Se reconoce a las personas privadas de la libertad los siguientes derechos: ...5) La atención de sus necesidades educativas, laborales, productivas, culturales, alimenticias y recreativas. Art. 201. El sistema de rehabilitación social tendrá como finalidad la rehabilitación integral de las personas sentenciadas penalmente para reinsertarlas en la sociedad, así como la protección de las personas privadas de libertad y la garantía de sus derechos. Art. 202. El Sistema se regirá por las siguientes directrices, literal 2, en los centros de rehabilitación social y en los de detección provisional se promoverán y ejecutaran planes educativos, de capacitación laboral, de producción agrícola, artesanal, industrial o cualquier otra forma ocupacional, de salud mental y física, y de cultura y recreación, literal 5. El estado establecerá condiciones de inserción social y económica real de las personas después de haber estado privadas de la libertad y Art. 203 ... 2) En los centros de rehabilitación social y en los de detención provisional se promoverán y ejecutarán planes educativos, de capacitación laboral, de producción agrícola, artesanal, industrial o cualquier otra forma ocupacional, de salud mental y física, y 
de cultura y recreación. (Asamblea Nacional del Ecuador, 2008).

\section{La educación en modelos penitenciarios}

Si bien es cierto que de una u otra forma durante el pasar de los años la educación ha tenido presencia en centros penitenciarios, no es menos cierto la necesidad de que su práctica conlleve al cumplimiento de derechos humanos, dignifique la tarea y se logre el objetivo principal de una reinserción a la sociedad de manera fortalecida, propositiva, emprendedora y ejecutable a través de un plan de vida altamente satisfactorio para la persona; ello lleva al cuestionamiento no de su existencia en sí dentro de los CRS sino de la interrogante del cómo llevar lo pedagógico, que aunque dentro de la estructura es solo una parte del proceso de reinserción es innegablemente una pieza fundamental para la rehabilitación de las PPL.

La preocupación por la realidad educativa en cárceles latinoamericanas ha sido motivo de estudios profundos y en un recorrido por ellos puede observarse en el común denominador de la contradicción institucional: por un lado los modelos, las leyes y políticas de Estado promueven la educación en las cárceles como un derecho ineludible para las personas privadas de libertad, pero por otro lado los contextos de encierro, el imaginario punitivo, más años de cárcel a la menor provocación, ha llevado a los centros de rehabilitación en Latinoamérica a un hacinamiento, insalubridad, falta de recursos y sobrepoblación descontrolada que impide el equilibrio entre lo que debe ser y la realidad (Rangel, 2013).

\section{Ecuador}

Una aproximación a la práctica pedagógica de centros penitenciarios en el Ecuador es motivo de estudio profundo que parte desde la visión filosófica del sistema penitenciario, donde el Código Orgánico Integral Penal garantiza una verdadera rehabilitación social, con el ejercicio de derechos y responsabilidades de las personas privadas de libertad, los CRS aseguran el desarrollo de capacidades para la reinserción social a través de políticas de gestión basados en la ética y dignidad humana (Zúñiga, 2014) en concordancia con el objetivo 4 del Sistema de Rehabilitación que señala "Reincorporar a la sociedad a quien haya cumplido con la sentencia condenatoria, debidamente rehabilitado y hacer el acompañamiento post carcelario" (Noriega, 2016).

El Ecuador bajo la implementación del modelo de gestión penitenciaria ha puesto énfasis en el eje de educación, cultura y deporte consciente de la importancia en el proceso de rehabilitación y reinserción de las PPL en el marco de sus derechos; la alfabetización, posalfabetización, educación básica, básica superior, bachillerato y superior se mantiene en coordinación permanente entre el Ministerio de Educación, la SENESCYT y el Ministerio de Justicia (Ministerio de Justicia, derechos humanos y cultos, 2017).

Los centros de rehabilitación social deben ser lugares en donde las PPL encuentren herramientas para construir una nueva forma de vida (Rovayo, 2015) para ello el proceso de inclusión social progresivo contempla de manera general las fases: 1) observación, 2) tratamiento y educación, 3) reinserción social con el fortalecimiento de vínculos familiares, 
lo que permita la operatividad desde la inducción con un diagnóstico integral siendo la evaluación educativa parte de ello, en cuanto al régimen progresivo la educación juega un papel fundamental que orienta la ejecución del plan de vida para que una vez cumplida la sentencia condenatoria en su plan de salida considere continuar con la formación profesional y trabajo (Ecuador, Ministerio de Justicia, Derechos Humanos y Cultos, 2013).

\section{Chile}

El sistema de este país contempla tres tipos de subsistemas: cerrado, abierto y postpenitenciario. El subsistema cerrado es para la población que ha sido privada de libertad, por medida cautelar de prisión preventiva, cumplimiento de una pena privativa de libertad o de una medida de apremio. En régimen semiabierto, recluidos en Centros de Educación y Trabajo (CET), y aquellos individuos condenados bajo un régimen abierto, es decir, con permiso de salida controlada, con libertad condicional, entre otros. El subsistema pospenitenciario se hace cargo de aquellos individuos que, una vez finalizado el cumplimiento de su pena, buscan oportunidades de reinserción social mayores. Existen evidencias de hacinamiento y sobrepoblación en las cárceles, donde la solución que ha propuesto el gobierno es la construcción progresiva de centros penitenciarios bajo convenios. (Sistema Carcelario y Libertad Condicional en Chile, 2016).

\section{Argentina}

La política penitenciaria del Gobierno argentino y también en materia de inclusión social considera la educación en contexto de encierro como una herramienta que permite a los internos comprender su historia personal y fijarse objetivos concretos para reinsertarse en la sociedad, bajo la posibilidad de crear un proyecto de vida propio; el Servicio Penitenciario Federal trabaja junto con el Ministerio de Justicia y Derechos Humanos y el Ministerio de Educación para una permanente oferta educativa de los establecimientos penitenciarios federales. E1 80\% de la población penal participa de algunos de los niveles de educación formal y no formal, así como de actividades culturales y talleres de capacitación. El proyecto educativo e interdisciplinario dentro de los establecimientos penitenciarios está ligado a programas de tratamiento para los internos, que les permiten alcanzar objetivos. La Dirección de Educación, Cultura y Deporte es el organismo técnico de ejecución y asesoramiento en el área y tiene por finalidad desarrollar y cumplir disposiciones en el área educativa y la organización pedagógica de la educación profesional (Argentina, Ministerio de Justicia y Derechos Humanos de la Nación, 2017).

Para Narciso (2012) es vital que los planteamientos políticos enfoquen como aspecto indispensable el tratamiento y cuidado de la emocionalidad de todos los actores que hacen a la institución, ya que si esta dimensión no es comprendida dificultosamente pueden hallarse nuevas propuestas dentro del pensamiento racional que condujo hasta esta realidad a la cárcel.

\section{Paraguay}

Elobjetivo del Centro de Estudios Penitenciarios en Paraguay tiene como eje fundamental la formación integral del personal (directivos, funcionarios, educadores, profesionales de 
salud, trabajo social) para laborar en este tipo de establecimientos, comprometidos con el precepto resocializador del sistema penitenciario y con las competencias y bases teóricas prácticas necesarias para una conducción idónea al interno de las cárceles (Paraguay. Ministerio de Justicia de la República del Paraguay, 2017).

\section{Colombia}

Un estudio diagnóstico realizado por el Gobierno de Colombia en cuanto a centros penitenciarios pone en evidencia que actividades de tratamiento penitenciario para la resocialización no tienen enfoque productivo y competitivo; se cuenta con escasa participación privada; existen debilidades en la articulación del modelo educativo para personas privadas de libertad con las políticas de educación nacional, limitación en la implementación del modelo educativo; baja interacción con los ámbitos familiar, comunitario y social en los programas de atención social y tratamiento penitenciario; y se presentan debilidades en los procesos de evaluación y tratamiento a la PPL condenados (Ministerio de Justicia y el Derecho, 2014).

Hay que resaltar que las condiciones de infraestructura son inadecuadas e insuficientes, desfavoreciendo la buena práctica de habilidades, competencias y comportamientos sociales fundamentales para la vida en libertad y para una correcta reinserción, con agravantes de obtención de libertad más por una redención de pena y sin reflexión alguna sobre la necesidad de plantear un proyecto de vida. Junto a lo expresado la deficiente de articulación de programas transversales con procesos de atención psicosocial y la baja participación por parte de los internos, dan cuenta de una realidad penitenciario lejana al ideal de una sociedad colombiana que busque disminuir cárceles en vez de crear y de rehabilitar en contrario de solo internar. (CONPES, 2015).

\section{Uruguay}

El modelo penitenciario en Uruguay plantea que las actividades educativas tienen tres objetivos: 1) Mantener a los internos ocupados provechosamente 2) mejorar su calidad de vida 3) conseguir un resultado útil (oficio, conocimientos, comprensión, actitudes sociales y comportamiento) que perdure más allá de la cárcel y permita el acceso al empleo o a una capacitación superior. Enfatizan como un punto importante en la consolidación de lo educativo en los contextos de encierro, la intervención de los Departamentos de Educación y Cultura: cuyo objetivo circunda en coordinar, planificar, organizar y administrar todas las acciones educativa-culturales, institucionales e interinstitucionales dentro del establecimiento. Así también se manifiesta la necesidad de generar espacios lo más similar a un contexto habitual educativo, para así naturalizar las actividades. La reinserción social y laboral de las PPL es el objetivo esencial dentro de todas las propuestas que se llevan a cabo (Uruguay. Ministerio del Interior. Instituto Nacional de Rehabilitación. Coordinación Educación y Cultura, 2014).

\section{Brasil}

En el literal g) del Manual de Tratamiento Penitenciario de Brasil, específicamente en la Asistencia en Terapia Ocupacional se busca proporcionar a los prisioneros 1) una formación profesional de iniciación y perfeccionamiento; 2) evaluación de rendimiento ocupacional y los 
componentes del desempeño ocupacional; 3) oficinas de orientación y capacitación para facilitar el aprendizaje de los participantes del taller; 4) la historia clínica de los datos de prisioneros en su área asignada; 5) la planificación, el seguimiento y la supervisión de las acciones relacionadas con la oferta y la ejecución de los trabajos a los presos; 6) planificación, dirección y ejecución de la atención, referencias, talleres terapéuticos y generación de ingresos, la rehabilitación y la reinserción social; 7) la recepción de los usuarios y sus familias y la humanización de la atención a la Educación, Salud, Trabajo y psicosocial; 8) desarrollo colectivo; 9) preparación de proyectos individuales terapéuticos y colectiva; 10) la realización de actividades de promoción de la salud y prevención de enfermedades para los presos y sus familias; 11) actividades relacionadas con su responsabilidad profesional. Y En el literal h) Asistencia Educacional incluye 1) la implementación de la política educativa en el Departamento Nacional de Prisiones - SPF en la Unidad; 2) el papel en la promoción y gestión de proyectos y sistemas de información dirigidas a los presos y sus familias; 3) participación en otras actividades en su área, en interés de la población reclusa (Brasil. Ministerio de Justicia y Ciudadanía, 2017).

\section{Perú}

Continuando con el recorrido de la educación según los modelos penitenciarios latinoamericanos, para Perú la educación en contexto de privación de libertad es aquella que brinda los establecimientos penitenciarios definido como un proceso de enseñanza-aprendizaje y que se desarrolla en un contexto socio cultural altamente complejo y especifico, a través del cual se asimilan y construyen conocimientos, capacidades y valores, aplicando metodologías y enfoques educativos multidisciplinarios en el tratamiento del interno; su objetivo principal es el de fortalecer en forma integral y multidisciplinaria el nivel de enseñanza aprendizaje de los internos en los establecimientos penitenciarios a través de capacitación y actualización docente de acuerdo al nivel y programa educativo... y en sus objetivos específicos expresa la necesidad de 1) Reeducar y capacitar a los internos a través de programas, proyectos y planes educativos; 2) Reinsertar a la sociedad a los internos que hayan cumplido y aprobado las acciones educativas para que puedan desempeñarse laboralmente en el Medio Libre; 3) Mejorar la metodología y técnicas de aprendizaje de los profesores de los establecimientos penitenciarios con asesoramiento y apoyo del Ministerio de Educación; 4) Sistematizar y uniformizar todas las acciones educativas que se desarrollan en todos los Centros de Educación Técnico Productiva (CETPRO) del país; 5) Gestionar la ampliación de servicios Educativos para los internos de los Establecimientos Penitenciarios dándoles formación Primaria y Secundaria de Adultos; 6) Promover la cultura y el arte en los establecimientos penitenciarios que permita sensibilizar a los internos y contribuya a mejorar su expresión y creatividad personal (Perú. Ministerio de Justicia. Instituto Nacional Penitenciario, 2017).

\section{Venezuela}

La información referente al sistema penitenciario en torno a la educación y procesos de reinserción es escasa, sin embargo, se encuentra regida por el Ministerio del Poder Popular para el Servicio Penitenciario y en su organigrama consta la Dirección General de Formación Educativa y 
Participación Protagónica (Venezuela. Gobierno

Bolivariano de Venezuela, 2017).

\section{Bolivia}

Las entidades de Servicios Penitenciarios Bolivianas brindan seguridad, atención integral y tratamiento a la población de los privados de libertad, haciendo de esta privación una oportunidad de construcción de proyectos de carácter personal y beneficio social, bajo un sistema de evaluación continua de los internos por su intervención en actividades de trabajo y formación; las actividades se clasifican en dos niveles: 1) prioritarias, encaminadas a cubrir necesidades inmediatas del privado de libertad como es el analfabetismo, alcoholismo, conductas sexuales desviadas, etc. y; 2) actividades complementarias. Los establecimientos cuentan con un catálogo de actividades formativas, culturales, deportivas, laborales, terapéuticas y asistenciales, disponibles en cada sección o módulo de los penales (Bolivia. Ministerio de Gobierno, 2017).

\section{Costa Rica}

En el informe de la Ministra de Justicia y Paz del 2015 se expresan los retos del sistema penitenciario nacional basado en estadísticas poblacionales, y a la conclusión emergente llega sobre políticas que permitan disminuir, redistribuir y atender a la sobrepoblación carcelaria por el hacinamiento como punto crítico del sistema (Sánchez, 2015), El componente de reinserción y educación no es abordado, tampoco se encontró información en la página oficial del Ministerio de Justicia y Paz del Gobierno de Costa Rica.

\section{Cuba}

El sistema penitenciario en Cuba es definido como el conjunto de normas generales, instituidas y delimitadas referidas a las penas en sí, el modo de su cumplimiento y el tratamiento de los penados y procesados. La Dirección de Establecimientos Penitenciarios del Ministerio del Interior es el encargado de garantizar el proceso de ejecución de la sanción de privación de libertad, de la sanción de trabajo correccional con internamiento, la medida de seguridad reeducativa de internamiento y la medida cautelar de prisión provisional. Dentro de sus procesos se busca: 1) adopción de un sistema progresivo avanzado y justo; 2) clasificación de la población penal que aseguran el óptimo tratamiento colectivo e individualizado; 3) participación voluntaria al trabajo socialmente útil y remunerado, con fines educativos y de asistencia y seguridad social para la familia; 4) ejecución de un subsistema educacional para la enseñanza general y técnica insertado en el sistema educacional gratuito del país; 5) atención médica y estomatológica primario y especializado, insertado en el sistema nacional de salud; 6) organización de actividades artísticas, culturales y deportivas (ECURED, 2017).

\section{E1 Salvador}

La Ley Penitenciaria de E1 Salvador en el capítulo IV de la educación e Instrucción, Art. 114 expresa que en cada centro penitenciario habrá una escuela en la que se impartirá educación básica, media, superior, técnica o universitaria a los internos, a la par se ejecutarán planes de estudio oficiales con el objetivo de al obtener su libertad puedan continuar con 
ellos; para su cumplimiento la administración penitenciaria, por medio del Ministerio de Justicia, podrá celebrar convenios o acuerdos con instituciones educativas, tecnológicas y universidades estatales o privadas. Interesante es el Art. 115 donde se manifiesta que aquellos internos que hubieren aprobado en forma satisfactoria la enseñanza básica y los que tuvieren una profesión o grado técnico que les permita contribuir con el régimen educacional dentro del centro, podrán participar como docentes o auxiliares (El Salvador, Ministerio de Justicia y Seguridad Pública, 2017).

\section{Guatemala}

En el Capítulo II de la Comisión Nacional de Salud Integral, Educación y Trabajo en los artículos del 69 al 72 denota los siguientes derechos acceder a: 1) servicio médico; 2) alimentación;3) podrá aspirar a un trabajo diurno en lugares cercanos a la cárcel, dependiendo de su estado legal; 4) uso de biblioteca, a estudios; 5) visitas familiares y conyugales; 6 ) a información, y a privacidad al momento de reunirse con el abogado a cargo de su defensa legal; 7) y a practicar cualquier confesión de fe (Gobierno de la República de Guatemala, 2016).

\section{Honduras}

En el reglamento General de la Ley del Sistema Penitenciario Nacional en su capítulo VI, el Art. 91 constan entre otros los objetivos del sistema de tratamiento penitenciario progresivo: 1) elaborar un programa de rehabilitación, reeducación y reinserción social, acorde a los principios de progresividad e individualización del tratamiento penitenciario; 2) contribuir con experiencias y metodologías alternativas o humanistaparticipativas, en la formación de valores y en el cambio de actitudes para un proyecto de vida digna de la persona interna; 3 ) generar procesos participativos en donde las personas internas puedan expresarse libremente, conociendo y valorando sus experiencias pasadas para construir un ambiente y futuro favorable a su desarrollo personal, familiar y comunitario; $y, 4$ ) desarrollar el Modelo de Tratamiento Penitenciario Progresivo en los establecimientos penitenciarios (Gobierno de la República de Honduras, 2015).

\section{México}

La Ley Nacional de Ejecución penal de México en su Capítulo IV expresa en los artículos $83-84$ - 85 - 86 los siguiente: 1) que la educación es el conjunto de actividades de orientación, enseñanza y aprendizaje, contenidas en planes y programas educativos, otorgadas por instituciones públicas o privadas que permitan a las personas privadas de su libertad alcanzar mejores niveles de conocimiento para su desarrollo personal; 2) la educación que se imparta en los Centros Penitenciarios será básica, media superior y superior mediante convenios con instituciones educativas del sector público, que les otorgarán la validez oficial correspondiente de los estudios culminados; 3) sus características serán: laica, gratuita, académico, cívico, social, higiénico, artístico, físico y ético, orientados en el respeto a la ley, las instituciones y los derechos humanos, será orientada por las técnicas de la pedagogía y quedará a cargo de profesores o maestros especializados; 4) las personas privadas de su libertad podrán acceder al sistema educativo con la finalidad de obtener grados académicos o técnicos y si obtienen una certificación por la autoridad educativa correspondiente podrán 
realizar las labores de docencia; 5) para personas indígenas, la educación que se les imparta será bilingüe y acorde a su cultura, para conservar y enriquecer sus lenguas, y la instrucción deberá ser proporcionada por maestros o profesores que comprendan su lengua; 6) los programas educativos serán conforme a los planes y programas oficiales que autorice la Secretaría de Educación Pública (Secretaría de Gobernación de México, 2016).

\section{Nicaragua}

La Ley del Régimen Penitenciario y Ejecución de la Pena en Nicaragua manifiesta al: 1) trabajo penitenciario, 2) capacitación, 3) actividades artísticas,4) actividades deportivas,5) instrucción general, como aspectos fundamentales para las personas privadas de libertad, cabe resaltar que en los centros penitenciarios se dispone de escuelas e institutos que alfabetizan, así también, brindan educación primaria y secundaria para los privados de libertad sobre la voluntad de los mismos; la educación es conforme a programas y políticas del Ministerio de Educación en convenios con universidades públicas $\mathrm{y}$ privadas, solo algunos centros penitenciarios impulsan la educación superior, ofertando carreras de: Agronegocios, Técnico Superior en Administración Agropecuaria, Farmacia, Administración de Empresas, Ingeniería civil, orientando a que puedan alcanzar los niveles académicos y títulos respectivos (Nicaragua. Ministerio de Gobernación de Nicaragua, 2014).

\section{Panamá}

En Panamá el acceso a la educación en el contexto penitenciario se desarrolla acorde a la estructura del Ministerio de Educación y sus lineamientos de 1) educación formal, 2) no formal, 3) informal, integrando procesos que promuevan aspectos culturales, recreativos, deportivos y espirituales; la educación formal académica es un proceso integral que abarca la alfabetización, la educación primaria hasta la educación secundaria y educación superior, así, una vez que obtenga la libertad se incorpore al mundo exterior con herramientas académicas que le brinden oportunidades de inserción laboral; por otro lado la educación no formal técnicalaboral, busca complementar, actualizar, y generar conocimientos académicos o laborales, sin estar sujetos a los niveles de la educación formal, su objetivo promueve perfeccionar a la persona en habilidades y destrezas para desempeñarse en actividades productivas (Panamá. Ministerio de Gobierno. Dirección General del Sistema Penitenciario, 2017)

Ante la situación penitenciaria latinoamericana es fundamental adoptar una postura teórica coherente y articuladora, basado en lo que Freire promueve sobre una educación con responsabilidad social, política y de compromiso moral hacia las personas que están en contextos de encierro, cuyos programas educativos aborden desde la educación formal, no formal y laboral, hacia la concreción del ideal ser humano en su proceso de reinserción a la sociedad (UNESCO, OEI, Gobierno de España, 2008). Varios son los estudios y propuestas por la preocupación de esta realidad en diversos países del mundo y que no es nada ajena a la realidad ecuatoriana, existe el modelo de pedagogía social ejecutoriado en países europeos desde los siglos XVIII y XIX (Pérez, 2002) tomándose como base para la actual propuesta del modelo de pedagogía social comunitario ejecutoriado hoy en día en 
España con fines de mejorar la calidad de vida de las PPL sustentado en dos pilares metodológicos: 1) acompañamiento psicosocial y educativo y 2) animación sociocultural incorporando el empoderamiento como motor de cambio (Morata, 2014).

Así, el tratamiento es un factor de suma importancia compuesto esencialmente de cuestiones socioeducativas orientados a reeducar y reinsertar a internos e internas sin excepción considerando programas individualizados que permitan la asistencia formativa, laboral o sociocultural (Fanny Añaños, 2013).

Para Francisco Serrano (2013) el ideal de una educación social penitenciaria se conduce desde una práctica de y hacia la libertad basada en principios paradigmáticos con visión humanista, metodológica y esencialmente liberadora, argumentando la actuación en los enfoques de modelos tales como: el participativo (Ayuso, 2000); Modelo para la autonomía (Pantoja, 2010); Modelos para el empoderamiento y/o de género (Juliano, 2010); Modelos del conocimiento de las personas y de las acciones pedagógicas (Saez, 2010); Modelos para la liberación (Caride, 2010). Hay que tomar en cuenta que los momentos educativos no se circunscriben tan solo al área educativa destinada, pues las diversas zonas de la prisión son contextos pedagógicos que permiten y viabilizan la interacción con el conocimiento y la producción de saberes de los estudiantes, monitores y docentes, el patio, la celda, aulas, zona de talleres, los espacios deportivos y recreativos son lugares educativos (García, 2009), un ideal que se practica condicionadamente dada la concepción de lo que estipulan los modelos penitenciarios a lo real, clasificaciones de personas privadas de libertad por niveles de peligrosidad, status, tiempo de condena y particularidades concretas de cada centro de rehabilitación social / prisiones, restringen acciones educativas en libertad proyectándose a procesos educativos y de reinserción limitados.

$\mathrm{Y}$ en este proceso los profesores son fundamentales en su formación de educadores con la articulación de varios elementos que conducen al análisis de un empoderamiento desde la perspectiva de quien aprende (personas privadas de su libertad), y las particularidades de los escenarios concretos donde se desarrolla la enseñanza, (en este caso los centros de rehabilitación social) (Diker 2008).

La tarea docente de manera general ya es compleja, pero lo es aún más la de educadores en las penitenciarías, pues sin duda alguna son actores principales en el proceso pedagógico. Varias interrogantes saltan a la mente de cómo es su tarea diaria en las cárceles? ¿qué propuestas desde sus competencias son abordadas?, temáticas necesarias al trabajar como profesores dentro de una prisión. Muchos de ellos no se han capacitado para esta labor y la percepción de soledad laboral contribuyen a la desidia o negación de trabajar desde una filosofía de educación liberadora en un contexto de encierro (Scarfó M. E., 2016).

Las competencias de un educador de contextos carcelarios se fundamentan en un perfil integral que contemple lo: 1) cognitivo: conocedor del contexto en aspectos filosófico, antropológico, sociológico, jurídico, comunicacional, cultural y pedagógico, conocedor del sujeto (jóvenes y adultos privados de libertad), capacidad para el diagnóstico, competencias comunicativas / 
creativas; 2) actitudinal: accionar sin prejuicios, sensibilizador social, con fuertes convicciones de un trabajo transformador, competencias empáticas; 3) procedimental: interventor pedagógico, animador sociocultural, promotor de trabajo, manejo didáctico con temáticas o problemáticas del afuera para un trabajo educativo en cárceles desde una perspectiva inter y multidisciplinaria (Scarfó I. A., 2007).

\section{Conclusiones}

El aspecto educativo a través del accionar del docente en centros penitenciarios hasta hoy en día y en la mayoría de sociedades ha sido una dura tarea invisibilizada y silenciosa, urgida de reconocimiento y valorización por parte de estamentos gubernamentales que desde la gestión y políticas públicas promueven su existencia más no articulan un espacio administrativo pedagógico dentro de jurisdicciones educativas, que oriente el perfil del educando a más de las especificidades elegidas por cada uno, al desarrollo de un perfil de competencias y capacidades generales que certifiquen su formación en derechos $y$ responsabilidades ciudadanas, competencias sociales y comunicativas, artísticas y de cultura con expresión creativa, destrezas tecnológicas, recreativas y deportivas, que aparezcan en su reflexión sobre actitudes y acciones cotidianas dentro y proyectadas a un futuro fuera del contexto de encierro.

\section{Trabajos futuros}

El proceso de reinserción social conlleva múltiples facetas a considerar tomando en cuenta el objetivo primordial de que la persona privada de libertad al momento de su incorporación a un contexto social libre, practique acciones sólidas y responsables desde la práctica en marcha de su plan de vida construido en tiempo de sentencia condenatoria; lo cual nos invita a analizar la necesidad de estudios técnicos de situaciones concretas y actuales del entorno carcelario bajo el modelo penitenciario en vigencia para cada país. En el Ecuador el modelo llama a la reflexión circundante a estándares de calidad de vida en el contexto de encierro; el proceso de construcción de un proyecto de vida profundo que repercuta positivamente al momento de reinsertarse a una vida social normal; necesidades pedagógicas desde el accionar de los miembros del sistema educativo dentro de centros penitenciarios; por lo que es evidente investigaciones y aportes en este aspecto relevante de toda sociedad en búsqueda de disminución de delitos y convivencias armónicas.

\section{Referencias bibliográficas}

Argentina, Ministerio de Justicia y Derechos Humanos de la Nación. (2017). Servicio Penitenciario Federal. Recuperado el 19 de 01 de 2017, de http://www.spf.gob.ar/www/educacion

Asamblea Nacional del Ecuador. (2008). Constitución de la República del Ecuador.

Añaños, F. (2013). Educación Social en Prisiones. Plkanteamientos Iniciales y Políticas encaminadas hacia la reinserción desde la perspectiva de género. Pedagogía Social. Revista Interuniversitaria, 22, 7-12. Obtenido de http://www. redalyc.org/articulo.oa?id=135031394001

Ayuso. (2000). La intervención socioeducativa en el tratamiento penitenciario. Pedagogía Social. Revista Interuniversitaria, 73 - 99. 
Bolivia. Ministerio de Gobierno. (2017). Situación de las Cárceles en Bolivia. Bolivia.

Brasil. Ministerio de Justicia y Ciudadanía. (2017). Ministerio de Justicia y Ciudadanía. Recuperado el 19 de 01 de 2017, de http://www. justica.gov.br/seus-direitos/politica-penal/sistema-penitenciario-federal-1/tratamento-penitenciario/tratamento

Caride, J. (2010). La Educación Social como práctica de y hacia la libertad en contextos penitenciarios. En Fanny T. Añaños (coord.), Las mujeres en las prisiones. La educación Social en contextos de riesgo y conflicto. 45 - 64 .

CONJIB. (2010). Declaración de San José. Recomendación la Conferencia de Ministros de Justicia de los Países. Obtenido de http://www. comjib.org/sites/default/files/DECLARACION-DE-SAN-JOSE.pdf

CONPES. (2015). Politica Penitenciaria y carcelaria en Colombia. Bogotá. Obtenido de https://colaboracion.dnp.gov.co/CDT/Conpes/ Econ\%C3\%B3micos/Pol\%C3\%ADtica\%20penitenciaria $\% 20 \mathrm{y} \% 20$ carcelaria.pdf

Constitución del Ecuador. (2008). Constitución de la República del Ecuador. Obtenido de http://www.asambleanacional.gov.ec/documentos/constitucion_de_bolsillo.pdf

Diker, G. (2008). La formación de maestros y profesores: Hoja de Ruta. Buenos Aires, Argentina: Paidos. Obtenido de https://docs.google.com/ viewer? $\mathrm{a}=\mathrm{v} \& \mathrm{pid}=$ sites\&srcid $=\mathrm{ZGVmYXVsd-}$ GRvbWFpbnx1bHNpbnZlc3RpZ2FjaW9uMnxneDo1ZGZ1ZGF1ZjI3NDE3NTU0
Ecuador, Ministerio de Justicia, Derechos $\mathrm{Hu}-$ manos y Cultos. (2013). Modelo de Gestión Penitenciaria del Ecuador.

ECURED. (2017). ECURED Conocimiento para todos y para todas. Recuperado el 19 de 01 de 2017, de https://www.ecured.cu/Sistema_Penitenciario_Cubano

El Salvador, Ministerio de Justicia y Seguridad Pública. (2017). Portal de Transparencia. Obtenido de http://www.dgcp.gob.sv/index.php/ temas/tema/leyes/leypenit

García, H. (2009). Colombia apuesta a un modelo educativo para la inclusión social de adultos en prisión. Convergence, 42(2 - 4), 173 - 185. Obtenido de https://search.proquest.com/docview $/ 877623036$ ? accountid $=36765$

Gobierno de la República de Guatemala. (2016). Ministerio de Gobernación, Dirección General del Sistema Penitenciario. Recuperado el 20 de 01 de 2017, de http://dgsp.gob.gt/wp-content/ uploads/2016/06/Reglamento.pdf

Gobierno de la República de Honduras. (12 de marzo de 2015). La Gaceta, Diario Oficial de la República de Honduras. Obtenido de http://www. tsc.gob.hn/leyes/REGLAMENTO_DE_LEY_ SISTEMA_PENITENCIARIO.pdf

Juliano, D. (2010). La criminalization de las mujeres. Estigmatización de las estrategias femeninas para no delinquir. En Fanny T. Añaños (coord.), Las mujeres en las prisiones. La educación Social en contextos de riesgo y conflicto. 25- 44. 
Ministerio de Justicia, derechos humanos y cultos. (2017). Ministerio de Justicia, derechos humanos y cultos. Recuperado el 06 de enero de 2017, de http://www.justicia.gob.ec/se-fortalece-el-eje-de-educacion-para-lograr-la-rehabilitacion-de-las-personas-privadas-de-libertad-del-pais/\#

Morata, T. (2014). Pedagogía Social Comunitaria: un modelo de intervención socioeducativa. Educación Social. Revista de Intervención SocioEducativa, 13-32. Obtenido de www.raco. cat/index.php/EducacioSocial/article/download/278526/368829

Narciso, L. (2012). Emergentes Identitarios En Cárceles de Santa Fe. AVA Revista de Antropologia, 20, 113-141. Obtenido de http://www.redalyc.org/pdf/1690/169031634006.pdf

Nicaragua. Ministerio de Gobernación de Nicaragua. (5 de agosto de 2014). Dirección General Sistema Penitenciario Nacional. Recuperado el 20 de 01 de 2017, de http:/www.migob.gob.ni/ spn/programas/

Noriega, M. (2016). Función Judicial. Obtenido de http:/www.funcionjudicial.gob.ec/www/ pdf/concursojuecesnotarios/materialdeapoyo/ SISTEMA\%20\%20PENITENCIARIO\%20 EN\%20EL\%20ECUADOR\%20Dr.\%20 Marco\%20Noriega.pdf

OEA. (2008). OEA Más derechos para más gente. Obtenido de CIDH : http://www.oas.org/es/ cidh/mandato/Basicos/PrincipiosPPL.asp

ONU. (1977). Naciones Unidas, Derechos $\mathrm{Hu}-$ manos. Obtenido de http://www.ohchr.org/SP/
ProfessionalInterest/Pages/TreatmentOfPrisoners.aspx

ONU. (1990). Naciones Unidas, Derechos $\mathrm{Hu}-$ manos. Obtenido de http://www.ohchr.org/ SP/ProfessionalInterest/Pages/BasicPrinciplesTreatmentOfPrisoners.aspx

Panamá. Ministerio de Gobierno. Dirección General del Sistema Penitenciario. (2017). Dirección General del Sistema Penitenciario. Recuperado el 2017, de Ministerio de Gobierno de Panamá: http://www.sistemapenitenciario.gob. $\mathrm{pa} /$ contenido/ofertas-de-estudio-y-trabajo

Pantoja, L. (2010). Las mujeres en las prisiones. La Educación Social en contextos de riesgo y conflicto de Fanny T.101-122.

Paraguay. Ministerio de Justicia de la República del Paraguay. (2017). Portal del Ministerio de Justicia. Recuperado el 19 de 01 de 2017, de http://www.ministeriodejusticia.gov.py/index. $\mathrm{php} /$ centro-de-estudios-penitenciarios

Pérez, G. (2002). Origen y Evolución de la Pedagogía Social. Pedagogía Social Revista Interuniversitaria, 193-231. Obtenido de Pérez Serrano, G; (2002). Origen y evolución de la Pedagogía Social. Pedagogía Social. Revista Interuniversitaria, () 193-231. Recuperado de http://www. redalyc.org/articulo.oa?id=135018332011

Perú. Ministerio de Justicia. Instituto Nacional Penitenciario. (2017). Instituto Nacional Penitenciario. Recuperado el 19 de 01 de 2017, de http://www.inpe.gob.pe/contenidos.php?i$\mathrm{d}=479 \&$ \&n $=32$ \&direccion $=1$ 
Rangel, H. (2013). Educación contra Corriente en las Cárceles Latinoamericanas: la enseñanza vs el castigo. Educação E̋ Realidade, Porto Alegre, 38(1), 15-32. Obtenido de http://www.scielo.br/ pdf/edreal/v38n1/03.pdf

Rovayo, V. (2015). Modernización y reformas al sistema penitenciario en Ecuador,. En D. H. Ministerio de Justicia, Memorias del Encuentro Regional: "Gestión y Administración Penitenciaria en el Contexto de los DD.HH. y la Seguridad Integral”. Quito, Ecuador: KLS, print. Recuperado el 5 de enero de 2017, de http:// www.justicia.gob.ec/wp-content/uploads/downloads/2015/11/Memorias-del-Encuentro-Regional.pdf

Saez. (2010). ¿Existe una educación específica para las mujeres en las prisiones? Algunas reflexiones desde la lógica profesional. En Fanny T. Añaños (coord.), Las mujeres en las prisiones. La educación Social en contextos de riesgo y conflicto. $101-122$.

Sánchez, C. (2015). Retos del Sistema Penitenciario Nacional. Rendición de Cuentas, San José, Costa Rica. Recuperado el 19 de 01 de 2017, de mjp.go.cr/doc/Download.aspx?Id=1108

Scarfó, F. (2007). Grupo de estudios sobre Educación en cárceles (GESEC). Decisio, 31 -36. Obtenido de http://tumbi.crefal.edu.mx/decisio/images/pdf/decisio_16/decisio16_saber5.pdf

Scarfó, F. (2016). Debates: sobre el rol de la escuela y de los educadores de adultos en las cárceles. 36(98), 99-107. Obtenido de http://www. scielo.br/pdf/ccedes/v36n98/1678-7110-ccedes-36-98-00099.pdf
Serrano, F. (2013). La educación social penitenciaria: ¿De dónde venimos y hacia dónde vamos?*/The prison social education: Wherefrom we come and towards where do we go? Revista Complutense de Educación, 24(1), 47 - 68. Obtenido de http://search.proquest.com/socialsciences/docview/1433250184/F12E9032C9D24007PQ/4? accountid=36765

Secretaría de Gobernación de México. (16 de 06 de 2016). Diario Oficial de la Federación. Recuperado el 20 de 01 de 2017, de http://dof. gob.mx/nota_detalle.php? codigo=5441664\&fecha $=16 / 06 / 2016$

SENPLADES. (2016). 9RC Vive la patria. Quito. Obtenido de http://www.planificacion.gob.ec/wpcontent/uploads/downloads/2016/01/9RC.pdf

Sistema Carcelario y Libertad Condicional en Chile. (13 de mayo de 2016). Libertad y Desarrollo, 1-7. Obtenido de http://lyd.org/ wp-content/uploads/2016/05/TP-1254SISTEMA-CARCELARIO-Y-LIBERTADCONDICIONAL-EN-CHILE-13-05-2016.pdf

UNESCO, OEI, Gobierno de España. (2008). Educación en prisiones en Latinoamérica. Derechos, Libertad y Ciudadanía. Brasilia. Obtenido de www.oei.es/historico/pdfs/ prisiones.pdf

Uruguay. Ministerio del Interior. Instituto Nacional de Rehabilitación. Coordinación Educación y Cultura. (2014). Encuentro Pensar y Hacer Educación en Contexto de Encierro. Durazno, Uruguay. Obtenido de https://inr. minterior.gub.uy/images/stories/pensar-yhacer-educacion.pdf 
Venezuela. Gobierno Bolivariano de Venezuela. (17 de 01 de 2017). Ministerio del Poder Popular para el Servicio Penitenciario. Recuperado el 2017, de http://www.mppsp.gob.ve/libraries/ organigrama_general.pdf
Zúñiga, L. (2014). Ministerio de Justicia. Obtenido de http://www.justicia.gob.ec/wpcontent/uploads/2014/05/c\%C3\%B3digo_ org\%C3\%A1nico_integral_penal_-_coip_ed._ sdn-mjdhc.pdf 